\title{
A Fiber-Optic Broadband CT/MR Video Communication System
}

\author{
H.K. Huang, R.H. Tecotzky, and Todd Bazzill
}

\begin{abstract}
Our department operates three magnetic resonance (MR) and three computed tomography (CT) scanners that are located in three different buildings up to $\mathbf{2 ~ k m}$ apart. We have designed and implemented a multichannel, fiber-optic broadband video communication system as a remote scanner monitoring network. This system consists of baseband and broadband fiberoptic transmitters, receivers, and multiplexers. The structure of the video network is supported by two strategically located headends (distributors) connecting local/remote scanners and monitoring stations. The system is capable of serving up to $5 \mathrm{~km}$ from each headend. The video signal from each scanner is sent through a baseband fiber-optic link to a headend, where it is frequency modulated, multiplexed with other scanner video signals, and distributed over broadband fiber-optic links to monitoring stations. Each receiver consists of a demodulator, a channel selectable tuner, and a video monitor. The current design provides up to 16 scanner channels and 16 remote monitoring station connections. Monitoring stations are placed in 14 clinical locations including the following reading rooms: thoracic, neuro, abdomen, musculoskeletal, gastrointestinal, genitourinary, and pediatric radiology. A radiologist can use any of these 14 monitoring stations to view a patient's CT/MR images in real-time as they appear on any of the six scanner consoles. By selecting the proper channel assigned to a patient's scanner, the radiologist may monitor the examination while using the telephone to communicate with the technologist at the scanner site. This fiber-optic broadband video communication system has been integrated into daily clinical use for over 6 months.

Copyright 11992 by W.B. Saunders Company
\end{abstract}

KEY WORDS: fiber-optic cable, broadband communication, video network.

B ROADBAND video communication can be used to monitor examinations of patients. We implemented a broadband coaxial

From the Medical Imaging Division, Department of Radiological Sciences, UCLA, Los Angeles, CA.

Supported in part by Public Health Service Grant No. RO1 CA 39063, RO1 CA 404565, and PO1 CA 51198, awarded by the National Cancer Institute, Department of Health and Human Services, and by the UCLA Department of Radiologi. cal Sciences Research Funds.

Address reprint requests to H.K. Huang, Medical Imaging Division, Department of Radiological Sciences, UCLA, Los Angeles, CA 90024-1721.

Copyright $(1992$ by W.B. Saunders Company

0897-1889/92/0501-0006\$03.00/0 video system for monitoring examinations performed in a magnetic resonance (MR) trailer located outside the hospital in 1987. ${ }^{1}$ Using this system, the technologist performs the examination at the trailer and transmits real-time MR images to the radiologist for immediate consultation. The radiologist views the images as the study is being performed and instructs the technologist to either continue, change sequence, or abort the study.

During the past 3 years, our department has expanded to three separate buildings and is operating three MR and three computed tomography (CT) scanners with two to follow. In order to facilitate the CT/MR operation, we have designed and implemented a fiber-optic broadband video system for clinical usage. ${ }^{2}$ This article describes the clinical environment, system design, and operation.

\section{CLINICAL ENVIRONMENT}

The Department of Radiological Sciences in our university hospital spans three buildings: Center for Health Sciences (CHS), Medical Plaza (MP), and the Taper Advanced Imaging Center. Center for Health Sciences is primarily in-patient; MP is primarily out-patient; and the Taper Advanced Imaging Center is used for research, in-patient, and out-patient MR imaging. The maximum distance between these building is about $2 \mathrm{~km}$. In CHS, there is one GE CT 9800 scanner (General Electric, Milwaukee, WI) in MP, there are two GE MR SIGNA scanners and two GE CT 9800 scanners; and in the Taper Advanced Imaging Center, there is a GE MR SIGNA scanner. In addition, one GE CT 9800 and one Siemens MR(0.6T) scanner (Siemens Medical Systems Inc, Iselin, NJ) are being installed. Figure 1 shows the relative locations of these three buildings. It is impractical for radiologists to travel to the three buildings to monitor CT/MR examinations daily. A multiplexed fiber-optic broadband video communication system is implemented to alleviate this problem.

The quality of the image in a broadband 


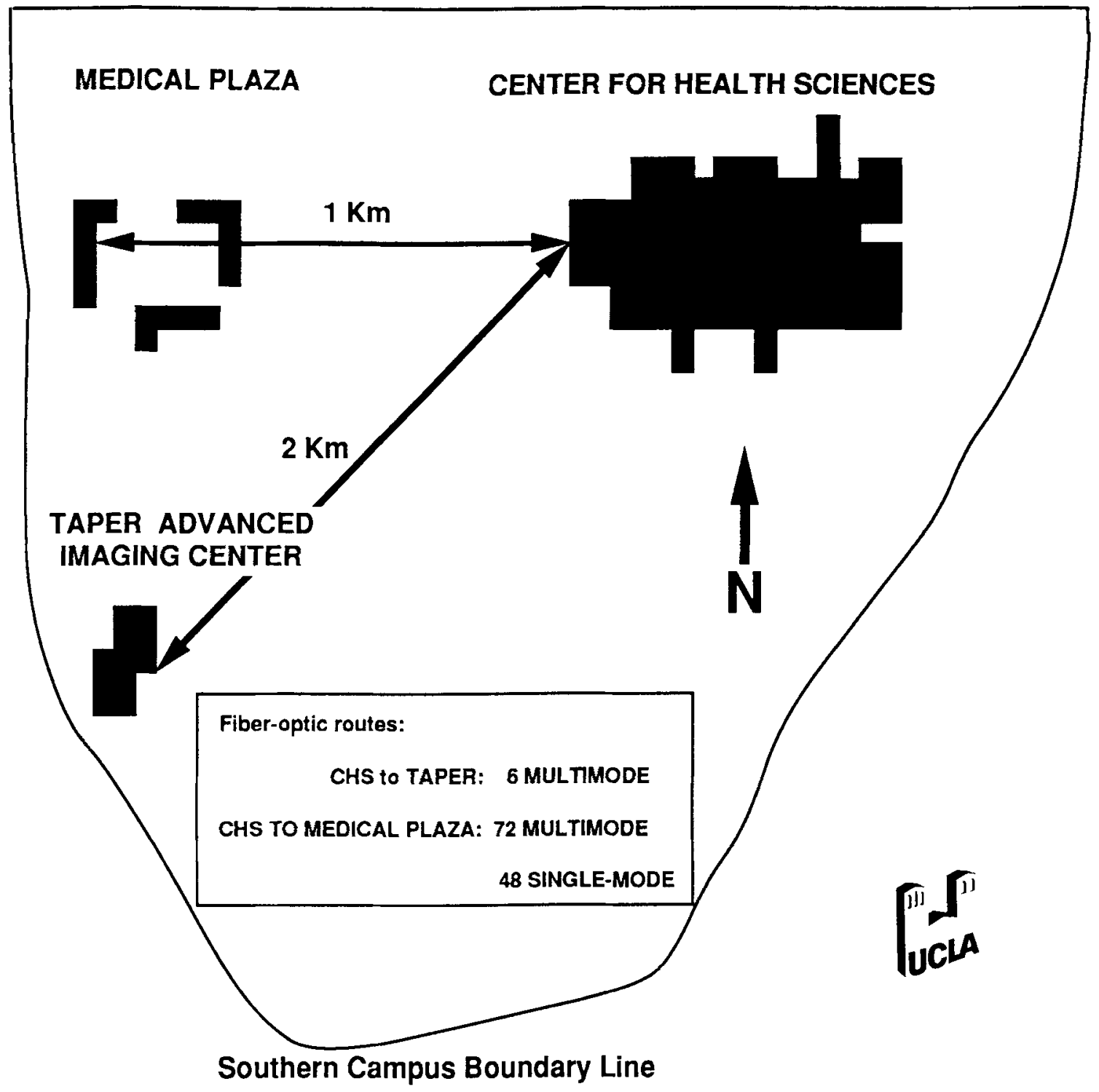

Fig 1. Relative locations of Taper Building (lower left), Medical Plaza (MP, upper left), and Center for Health Sciences (CHS, upper right).

communication system, using coaxial cables as the transmission media, begins to deteriorate when the distance between the headend and any image source or receiver is over $0.3 \mathrm{~km}$. Therefore, conventional coaxial cable broadband system cannot be used to connect these scanners.

In July 1990, we completed the installation of a system of fiber-optic cables. The two PACS (picture archiving and communication system) Hub Rooms, one in CHS and the other in MP, contain patch panels with 72 multimode $(62.5 /$ 125 microns) and 48 single-mode (8.3/125 mi- crons) fibers dedicated for their connection between CHS and MP for our departmental use. Six dedicated multimode fiber-optic cables connect the Taper Advanced Imaging Center to the UCLA Campus Fiber-Optic Backbone and then are routed to the CHS PACS Hub Room. Singlemode fiber optic cables are routed from each PACS Hub Room to many local/remote reading rooms. This comprehensive fiber-optic system allowed us to design and implement our broadband video system. The purpose of using fiber-optic transmission is to take advantage of the low signal loss in the medium. ${ }^{3-5}$ 


\section{MODELING}

In order to design the fiber-optic broadband video network, we first derived a model to describe the signal loss characteristics due to various components in the communication system. The model SL $=F(B, D, M, C, W, T R)$ describes the signal loss ( $\mathrm{SL}$ ) of the image as a function of the image bandwidth (B), the distance between two nodes (D), the mode of the fiber used (M), the fiber connector type (C), the wavelength of the light used $(\mathrm{W})$, and the characteristics of the optical transmitter/receiver pair (TR). As a first approximation, we assume $F$ is a linear combination of all these variables.

In this model, the losses associated with $\mathrm{D}$, $\mathrm{M}, \mathrm{C}$, and $\mathrm{W}$ can be measured through the existing fiber-optic cables using an Optical Time Domain Reflectometer (OTDR). The CT/MR images have a known bandwidth (B), which is approximately $25 \mathrm{MHz}$ with a horizontal scan rate of $33.36 \mathrm{KHz}$ (1,120 lines). We estimated the total video signal loss (SL) acceptable to be $-20 \mathrm{~dB}$ based on visual inspection. From this information we designed the optical transmitter/ receiver pair (TR) required for this system. Unfortunately, the current "off-the-shelf" optical transmitter/receiver pair available can only accommodate a $10 \mathrm{MHz}$ video signal bandwidth. Therefore, we had to reduce the bandwidth of the transmitted video signal from 25 $\mathrm{MHz}$ to $10 \mathrm{MHz}$ in two stages. First, the video signal was reduced from $25 \mathrm{MHz}$ to a $10 \mathrm{MHz}$ video signal, the $10 \mathrm{MHz}$ video signal was then transformed to optical signal for transmission. This was accomplished by using a fiber optic laser transmitter tuned to $10 \mathrm{MHz}$ (X285TVV5, Meret, Inc, Santa Monica, CA). The result is equivalent to pass the image through a lowpass filter, hence, a minor degradation of the image quality.

\section{THE VIDEO COMMUNICATION SYSTEM}

The multiplexed broadband video communication system is an established image transmission method. The term multiplexed refers to the method of modulating and combining multiple baseband (a single video) signals into a composite broadband signal; effectively using a single broadband (relatively wide bandwidth) channel to transmit multiple baseband (relatively narrow bandwidth) channels.
Real-time video images are transmitted from CT/MR scanners to one of the headends, where they are converted to specific ranges of radio frequencies, and distributed to receivers in a similar manner as in cable television technology. The communication medium used is either a multimode (shorter distance) or a singlemode (longer distance) fiber-optic cable. The $512 \times 512 \times 8$ pixel image (CT) and $256 \times$ $256 \times 8$ pixel image (MR) can be assigned to a given channel (10 MHz in bandwidth) in the 60 to $660 \mathrm{MHz}$ frequency range. The video signal at the scanner is converted from an electrical to an optical signal before it is transmitted to a headend. When the video signal is received at a headend, it is converted back from an optical to an electrical signal. Here, it is multiplexed (modulated \& combined) with the other scanner video signals and signals from the other headend, creating a multiplexed, broadband video signal.

Next, the broadband signal is converted from an electrical to an optical signal and is routed to an optical splitter where the signal is distributed to all monitoring stations. Here at the monitoring station, the optical broadband signal is converted back to an electrical signal, demodulated (channel is selected), and displayed on a viewing monitor. Each monitor station consists of an optical receiver, a demodulator (channel selector), and a modified CONRAC 1023 line, progressive scan, 8-bit video monitor (Duarte, CA).

Our current configuration allows us to transmit images from as many as 16 scanners to 16 remote monitoring stations. Monitoring stations are placed in 14 clinical locations including the following reading rooms: thoracic, neuro, abdomen, musculoskeletal, gastrointestinal, genitourinary, and pediatric radiology. Figure 2 shows the block diagram of the video communication system. Table 1 shows the cost estimation of our system, which consists of connections to six scanners, 14 display stations, and two headends-one at CHS and the other at MP. The actual price for a production system should be lower since our system was a prototype design. This cost does not include the fiber optic cables installation.

\section{CLINICAL OPERATION}

In any of the 14 locations, a radiologist, by selecting the proper channel assigned to the 


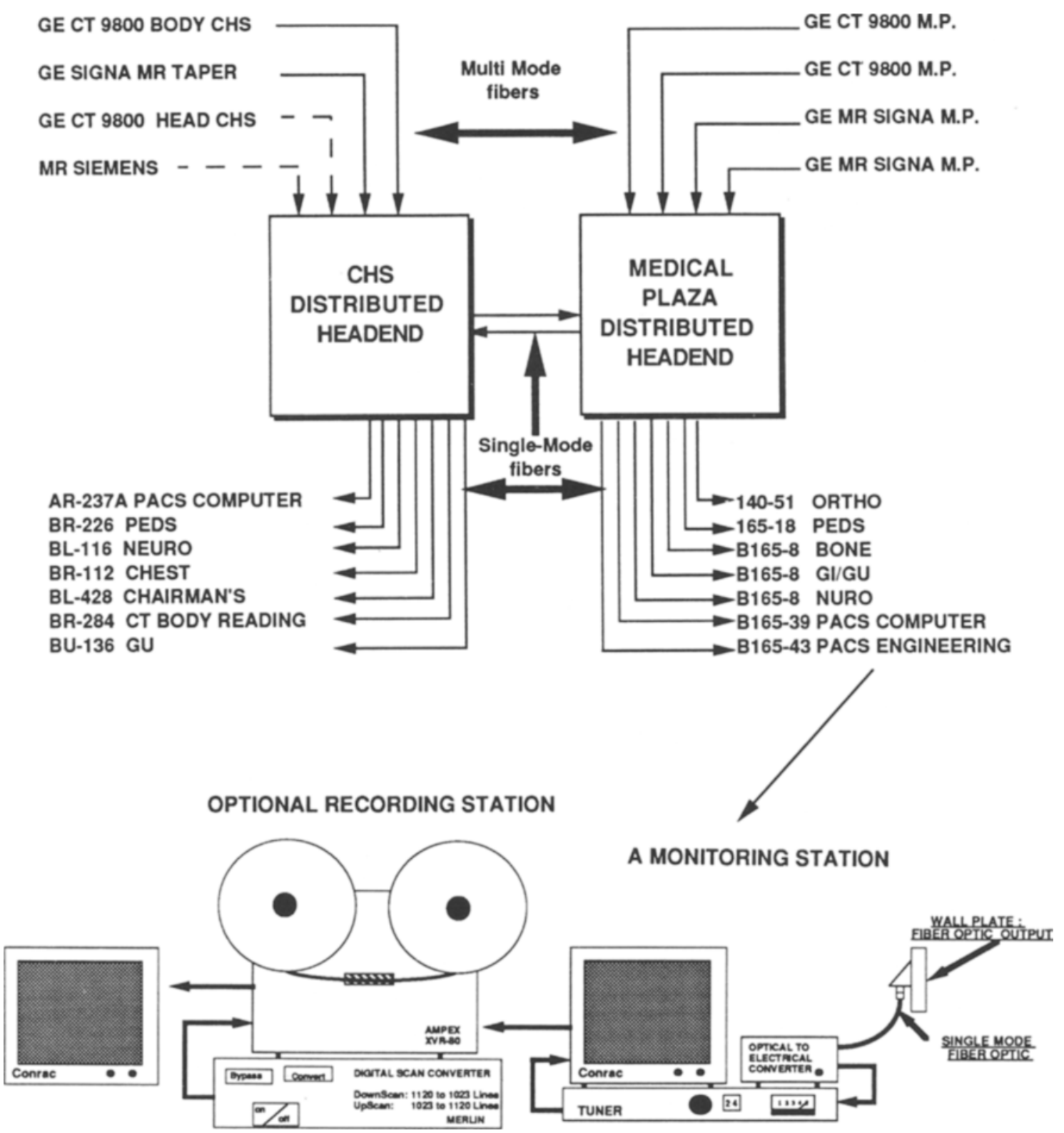

Fig 2. Multi-channel fiber optic broadband CT-MR patient monitoring system. The Block diagram of the fiber optic broadband video system which connects three $C T$ and three MR scanners to two headends and distributes images to 14 monitoring stations.

scanner, can view a patient's CT/MR examination in real time while the study is being performed. The radiologist can communicate with the technologist by a telephone to monitor the examination. The system has been in clinical operation 24 hours a day, 7 days a week since November 1990. After 6 months of fine tuning, it is very reliable and requires minimal maintenance. Preventative maintenance is performed by a PACS technologist daily, which includes
Table 1. Estimated Costs of the Fiber-Optic Broadband System

\begin{tabular}{lrrr}
\hline \multicolumn{1}{c}{ Equipment } & Unit Price (\%) & Units & \multicolumn{1}{c}{ Total } \\
\hline Laser transmitter (one/scanner) & 1,700 & 6 & 10,200 \\
Headend (distributor) & 22,000 & 2 & 44,000 \\
Receiver (one/station) & 4,500 & 14 & 63,000 \\
Monitor (one/station) & 2,000 & 14 & 28,000 \\
Total & & & 145,200 \\
\hline
\end{tabular}

NOTE. This estimation is for six scanners, 14 display stations and 2 headends, one at each building. 
visual checks on both headends and each video monitoring station for image quality.

\section{CLINICAL USAGE SURVEY}

After 6 months of operation, we conducted a clinical usage survey of this on-line monitoring system for remote CT/MR scanners. The results of the compiled data show significant usage of the system by a wide spectrum of radiologists. The system has been accepted into the clinical environment as a highly valued diagnostic tool. In the survey, we polled 32 radiologists including residents and fellows. The system's performance was evaluated in five categories: image quality, speed of display, ease of use, reliability, and overall impression. Table 2 shows the results from the survey. We also surveyed the reasons for using this video communication system by the radiologists. Table 3 shows the results.

From the results of our survey, we conclude that the system's performance is excellent and it is used as a time saving tool for the radiologist. Furthermore, the system saves the radiologist time through better communication and fewer delays; thus, the quality of patient care is

Table 2. Fiber-Optic Video Communication System Performance: A User Survey

\begin{tabular}{|c|c|c|c|c|c|c|c|}
\hline & & Poor & Fair & Good & Excellence & Mean & $\begin{array}{l}\text { Standard } \\
\text { Dev }\end{array}$ \\
\hline \multirow[t]{2}{*}{ Image quality } & $\#$ & 0 & 1 & 15 & 16 & 3.47 & 0.57 \\
\hline & $\%$ & 0 & 3.1 & 46.9 & 50.0 & & \\
\hline \multicolumn{8}{|c|}{$\begin{array}{l}96.9 \% \text { of the radiologists surveyed said that the image quality } \\
\text { of the system is good or excellent. }\end{array}$} \\
\hline \multirow[t]{2}{*}{ Speed of display } & $\#$ & 0 & 3 & 17 & 12 & 3.28 & 0.63 \\
\hline & $\%$ & 0 & 9.4 & 53.1 & 37.5 & & \\
\hline \multicolumn{8}{|c|}{$\begin{array}{l}90.6 \% \text { of the radiologists surveyed said that the speed of } \\
\text { display of the system is good or excellent. }\end{array}$} \\
\hline \multirow[t]{2}{*}{ Ease of Use } & $\#$ & 1 & 3 & 16 & 12 & 3.22 & 0.75 \\
\hline & $\%$ & 3.1 & 9.4 & 50.0 & 37.5 & & \\
\hline
\end{tabular}

$87.5 \%$ of the radiologists surveyed said that the "ease of use" of the system is good or excellent.

\begin{tabular}{|c|c|c|c|c|c|c|}
\hline \multirow[t]{2}{*}{ Reliability } & $\# 0$ & 4 & 14 & 12 & \multirow[t]{2}{*}{3.06} & \multirow[t]{2}{*}{1.05} \\
\hline & $\% \quad 0$ & 12.5 & 43.7 & 37.5 & & \\
\hline \multicolumn{7}{|c|}{$\begin{array}{l}81.2 \% \text { of the radiologists surveyed said that the reliability of } \\
\text { the system is good or excellent. }\end{array}$} \\
\hline \multirow[t]{2}{*}{ Overall impress } & $\# 0$ & 2 & 18 & 12 & 3.31 & 0.59 \\
\hline & $\% \quad 0$ & 6.2 & 56.2 & 37.5 & & \\
\hline
\end{tabular}

NOTE. Scale: 1 (poor) to 4 (excellent).
Table 3. Reasons for Using the Video Communication System: A User Survey

\begin{tabular}{lc}
\hline \multicolumn{1}{c}{ Reasons for Using the System } & Percentage of Usage \\
\hline Consultation & 51.6 \\
Saves time & 87.1 \\
Helped in daily practice & 87.1 \\
Occasional primary diagnosis & 32.3 \\
Multiple/simultaneous case monitoring & 32.3 \\
Recording images on video tape would be & \\
$\quad$ useful & 32.3 \\
\hline
\end{tabular}

improved. During the first 6 months, system reliability had become a minor concern due to a faulty component in the headend. We have since modified the design, and the system uptime has reached nearly $100 \%$.

\section{DISCUSSION}

Two advantages of this fiber optic broadband communication system are that it is inexpensive and it is capable of transmitting images from many sources in real time. The system can be used primarily as a CT/MR examination monitoring system. The system also has some disadvantages. First, the image quality may not be sufficient for primary diagnosis because the image transmitted to the video monitor is of a lower bandwidth. Second, images appearing on the monitor are volatile and therefore cannot be stored or retrieved later.

In order to have a temporary image archival, we have completed the interface design of this system to a high resolution video tape recording system (AMPEX XVR-80, San Fernando, CA) studio recorder, San Fernando, CA. This video recorder coupled with a Merlin Engineering's DownScan Converter (Palo Alto, CA), can be connected to any of the 14 monitoring stations to record images from any of the six scanners (Fig 2 lower left). The resolution of the images saved in this video recorder is expected to be equivalent to what appeared on the monitor. In the next few months, we will integrate this recorder with the broadband system by placing it in a centralized location in the department. This recorder will be used as a short-term ( 1 to 2 days) archival system for an emergency or a quick review purpose.

\section{ACKNOWLEDGMENT}

We thank Dr Jim Sayre for designing the user survey and Jim Tagawa and Sandy Eldredge for conducting the survey. 


\section{REFERENCES}

1. Huang HK, Lou SL, Cho PS, et al: Radiological image communication methods. AJR 155:183-186, 1990

2. Huang HK, Stewart BK, Loloyan M, et al: Radiological image communication with fiberoptic media. Radiology, 177(P), P321, 1990

3. Olshansky R, Lanzisera VA: 60-channel FM video subcarrier multiplexed optical communication system. Electronic Letters 23:1196-1198, 1987
4. Way WI: Multi-channel FM video transmission using traveling-wave amplifier in $1300 \mathrm{~nm}$ for subscriber distribution. Electronic Letters 24:1370-1372, 1988

5. Darcie TE, Bodeep GE: Lightwave subcarrier CATV transmission systems. Institute of Electrical and Electronic Engineers Trans Microwave Theory Tech vol MTT-38, 1990, pp 524-533 